\title{
CFD Analysis and Optimization of Effect of Shroud with Multi-outlets on Airflow Uniformity in a Frost-Free Refrigerator
}

\author{
X. F. Du ${ }^{1}$, C. Y. Zong ${ }^{2}$, Q. D. Fu ${ }^{1}$ and J. R. Zhang ${ }^{1 \dagger}$ \\ ${ }^{I}$ School of Mechanical Engineering, Southeast University, Nanjing 211189, China \\ ${ }^{2}$ School of Mechanical Engineering, Dalian University of Technology, Dalian 116023, China \\ $\dagger$ Corresponding Author Email: zhangjr@seu.edu.cn
}

(Received November 3, 2019; accepted June 8, 2020)

\begin{abstract}
The shroud is a key component of the frost-free refrigerator and its geometric parameters have great influence on the aerodynamic performance of the whole system. Previous researches mainly focused on the effect of other components, such as the fan, shelves, or plate-evaporator. In this paper, the influence of the shroud with multi-outlets on the flow distributions of a frost-free refrigerator is studied thoroughly with the help of Computational Fluid Dynamics (CFD) tools. A 1/2 3-D CFD model is developed, where the verification of turbulence models and mesh independence tests are performed by comparing the mass flow rate obtained by different model configurations. The standard $k$-epsilon is deemed as the most suitable turbulence model choice and a mesh with Fine level is considered as mesh independence. To obtain the boundaries of the developed CFD model, an airflow velocity test rig is built and constructed. To convert the measured data to CFD model, Structural Response Vector (SRV) method is implemented for velocity profile fitting, and the fitted surface is assigned by User Defined Functions (UDF) macros in simulations. A series of simulations are carried out with the developed model, and the results indicates that no streamline in the middle two cavities of the original freezer compartment and the airflow velocities at the three outlets of the investigated shroud show a certain difference. To optimize the flow distribution, the agent model based on the BP neural network is established, in which four critical parameters of the shroud are adopted as design variables. The results show that the velocity streamlines in the middle two cavities are significantly increased after optimization and the value of the mean square error model constructed in optimization has a reduction of $61.09 \%$ compared with the original design.
\end{abstract}

Keywords: Shroud; CFD simulation; Test rig; Airflow uniformity; Optimization.

\section{NOMENCLATURE}

$\mathrm{d}_{1} \quad$ width of the outlet 1

$\mathrm{d}_{2} \quad$ distance between the edge of the

$\mathrm{J}_{1} \quad$ mean square error of the airflow velocities of the three outlets

$\mathrm{J}_{2} \quad$ mean square error of the airflow velocities at five points on the centerline of the freezer compartment

MAE Mean Absolute Error

MAX Maximum Absolute Error
$\mathrm{Q}_{1} \quad$ flow rate of outlet 1

$\mathrm{Q}_{2} \quad$ flow rate of outlet 2

Q3 flow rate of outlet 3

$\mathrm{R} \quad$ radius of the arcs at the outlets 2 and 3

$\mathrm{R}^{2} \quad$ complex correlation coefficient

RANS Reynolds Averaged Navier-Stokes

RMSE Root Mean Square Error shelves to the edge of the vertical board

SRV Structural Response Vector

\section{INTRODUCTION}

The frost-free refrigerator relies on a system consisting of an axial fan and a shroud in the freezer compartment and an evaporator hidden behind the

wall of the freezer compartment to drive the cold air to circulate and achieve the cooling effect. It has the advantages of automatic defrosting and fewer odors, which make it popular with consumers. Since the air circulation in the freezer compartment of the 
frost-free refrigerator depends on the joint action of the shroud and the fan, the problem of uneven temperature distribution and excessive noise in the freezer compartment is mainly related to the unreasonable design and installation position of the shroud and the fan. Shrouds in different refrigerators are diverse, and their influence on the flow field in the compartment is very significant. Especially for the frost-free refrigerator containing large volume freezer compartments, there are often multiple air outlets and curved regions with large curvature for the shroud, which will easily lead to unreasonable air distribution at each outlet and large turbulence or backflow through the curved areas of the shrouds. Those above phenomena directly affect the refrigeration effect of the refrigerator and may generate large aerodynamic noise. Therefore, it is significant to analyze the influence of the geometric parameters and the positon of the shroud on the flow field in the freezer compartment and carry out structural optimization to achieve better aerodynamic performance.

The air or liquid flow duct generated by shrouds directly affects the trajectory of the fluid and the key parameters of fluid dynamics such as flow rate, static pressure or temperature distribution. A fluid system including a poorly designed shroud could not produce the flow and temperature fields that we need. What's more, significant turbulence or noise could be produced in specific areas or locations in that fluid system, which will severely affect the fluid dynamics performance of products. In the past two decades, many researchers have conducted studies for fluid systems containing shrouds using CFD simulation or experimental methods. Early numerical simulations and experimental studies have shown that shroud can increase the flow rate and efficiency of axial fans compared to the fans without shroud (Neal et al. 2007 and Abe et al. 2005). Hu et al. (2011) conducted an experimental study on the effect of the shroud on the performance of the cooling fan of the radiator installed at the front of the car and found that the vorticity and turbulence intensity of a fan with a shroud is lower than without a shroud.

In order to deeply analyze the influence and mechanism of the parameters and position of the shrouds on hydrodynamic performance of the fluid system, the CFD numerical simulations and experimental methods are used to carry out analysis and optimization of the systems containing shrouds in many application fields. In the field of household appliances, Wu et al. (2014) studied the effects of parameters of the bell-shaped shroud on the aerodynamic performance of DC frequency conversion split-type air-conditioning units based on three-dimensional computational fluid dynamics (CFD). Huang et al. (2017) used computational fluid dynamics and experimental methods to study the effects of different shroud structures on the aerodynamic performance of an axial fan at the refrigerator compressor. Hu et al. (2006) used CFD to study the influence of the shroud on the aerodynamic performance of the split-unit airconditioning outdoor unit and verified that the shroud with diversion ducts can improve the aerodynamic performance. Ren et al. (2013) investigated the air flow rate and radiation noise of the shroud with an axial fan in the mechanical room of the household refrigerator and found that the length of the shroud and the serrated structure contribute significantly to the flow velocity and radiation noise.

In other application fields, Shibata et al. (2019) and Duan et al. (2019) used computational fluid dynamics (CFD) analysis and experimental tests to study the turbine rotor shroud and discussed the effect of the axial distance between the cyclone breaker and the rotor shroud on efficiency. Aranake et al. (2015) studied the flow physics and performance of a shrouded turbine by solving the Reynolds Averaged Navier-Stokes equation to supplementing the transition model. Other studies of turbine systems including shrouds have focused on the effects of shrouds on blade performance and their effects on the flow field and system performance (Abu-Thuraia et al. 2018, Jiang et al. 2014 and Jung et al. 2016). Mansouri et al. (2018) studied the effects of relative shroud motion on heat transfer and secondary flow near the tip of transonic turbine blades by using the finite volume method. Yalcin et al. (2008) used the finite volume CFD method to study the effect of the gap change between the tip of the fin and the shroud on the steady-state heat transfer. Zhou et al. (2013) investigated the influence of the impeller rear shroud radius on the axial force and pump hydraulic performance of a multi-stage deep-well centrifugal pump (DCP). Pan et al. (2018) studied the effects of shrouds with different coverage positions on the hemodynamics and hydraulic performance of blood centrifuge pumps based on CFD.

In terms of the influence mechanism of shrouds, Wadia et al. (2008) compared the aerodynamic performance of a partial span shroud and a shroudfree fan blade in the first stage of a multi-stage fan of an aircraft engine. Jafari et al. (2014a) proposed that the sub-atmospheric back pressure is the main mechanism for the increase in drive power and they also found that sub-atmospheric back pressure is the most influential factor in a power increase of a small commercial wind turbine (Jafari et al. 2014b).

Previous studies on refrigerators have focused on the shelves (Zhang et al. 2014 and Avc1 et al. 2016), the gap between the partition and inner wall or door of the refrigerator (Ding et al. 2004), the plate-evaporator (Belman-Flores et al. 2016), and the new heat exchange material (Elarem et al. 2017). However, little research has been done on the influence of complex shrouds on the flow fields in refrigerators. The global performances of the complex shroud with multi-outlets featuring different geometric parameters and relative distances need to be investigated further.

In this paper, the effects of the shroud shape with multiple air outlets, the width of the outlets and the relative position of the shroud to the axis fan on airflow uniformity in the frost-free refrigerator are investigated. This paper is organized as follows, 
Section 1 is the introduction and Section 2 is the description of the refrigerator. In Section 3, we constructed an experimental platform to measure airflow velocities. Section 4 is devoted to the CFD analysis of the fluid domain in the freezer compartment. Section 5 exposes through optimization of the shroud and other geometric parameters to improve aerodynamic performance. Conclusions close the paper in Section 6.

\section{DETAILS OF THE REFRIGERATOR}

The structure of the frost-free refrigerator investigated in this paper is shown in Fig. 1. The size parameters of the refrigerator are $1722 \mathrm{~mm}$ (height) by $910 \mathrm{~mm}$ (width) by $610 \mathrm{~mm}$ (length). The refrigerator consists of a freezer compartment and two cold storage compartments, in which the freezer compartment on the left and the two cold storage compartments on the right. The cooling method in the freezer compartment of the refrigerator is air-cooled and a pneumatic system consisting of an axial fan and a shroud is located above the evaporator. The rotation of the axial fan drives the air to flow through the evaporator, and heat exchange occurs on the surface of the evaporator to lower the air temperature. Then the cold air flows out through the three outlets of the shroud and enters the freezer compartment to cool the foods. There are three shelves in the freezer compartment for placing items and the size parameters of the freezer compartment are 1591 $\mathrm{mm}$ (height) by $546 \mathrm{~mm}$ (length) by $272 \mathrm{~mm}$ (width).

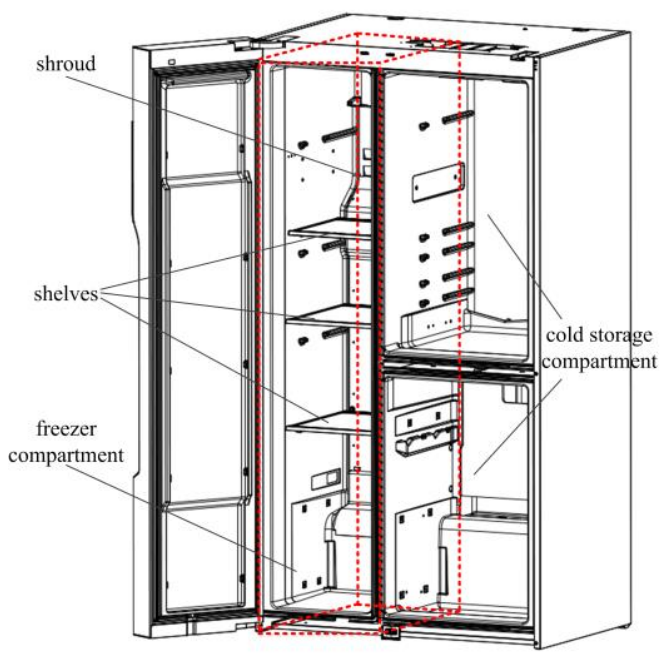

Fig. 1. The overall structure of the refrigerator.

\section{TEST Rig OF AiRfLOW VELOCITY}

\subsection{Composition of the Pneumatic System}

The pneumatic system consisting of the shroud, the axial fan and the back wall of the freezer compartment is shown in Fig. 2. The axial fan has an outer diameter of $100 \mathrm{~mm}$, a number of blades of 4, an axial height of $25 \mathrm{~mm}$, a hub ratio of 0.25 and a rotational speed of $2504 \mathrm{r} / \mathrm{min}$. The height of the shroud in the vertical direction is $314 \mathrm{~mm}$, the width and thickness of the shroud is $272 \mathrm{~mm}$ and $2.5 \mathrm{~mm}$, and the angle between the inclined portion of the shroud and the vertical direction is $52^{\circ}$. There are three outlets on the shroud. The size of the air outlet 1 is $232 \mathrm{~mm}$ by $11 \mathrm{~mm}$, and the sizes of the air outlets 2 and 3 are 232 by $8 \mathrm{~mm}$. When the axial fan rotates, the air flows from the bottom to the top, and flows out from the three outlets of the shroud into the freezer compartment. In the freezer compartment, the cold air flows from top to bottom, and the food on the shelves is cooled by the cold air. When the air flows to the lower part of the freezer compartment, it enters the area below the axial fan through the opening of the vertical partition and will be driven to flow out again from the three outlets of the shroud, thereby achieving continuous circulation in the freezing compartment.

\subsection{Airflow Velocity Measurement}

To determine the initial condition of the following CFD simulation, an experimental platform is conducted in this paper to measure the airflow velocity on the plane which is $12 \mathrm{~mm}$ above the axial fan in the freezer compartment of the refrigerator. The position of the measuring points is shown in Fig. 3. The circular points in Fig. 3 are measuring points, and six diameters at angles of $0^{\circ}$, $30^{\circ}, 60^{\circ}, 90^{\circ}, 120^{\circ}$ and $150^{\circ}$ to the width direction of the refrigerator were selected as measurement lines. The diameter of the measuring surface is $105 \mathrm{~mm}$, which is slightly larger than the outer diameter of the axial fan. Each measuring line is equidistantly distributed with 11 measuring points with a spacing of $10.5 \mathrm{~mm}$. The measuring points on each measuring line are numbered, and the point number increases from 1 to 11 with the direction indicated by the arrow next to each measuring line in Fig. 3. The airflow velocity measurement experiment site and the instrument used are shown in Fig. 4. The model of the anemometer used in the experiment is TENMARS TM-4002 with a measuring range of $0.01 \sim 25 \mathrm{~m} / \mathrm{s}$, a resolution of $0.01 \mathrm{~m} / \mathrm{s}$ and an error range of $\pm 3 \%$ measured value $\pm 1 \%$ full-scale value.

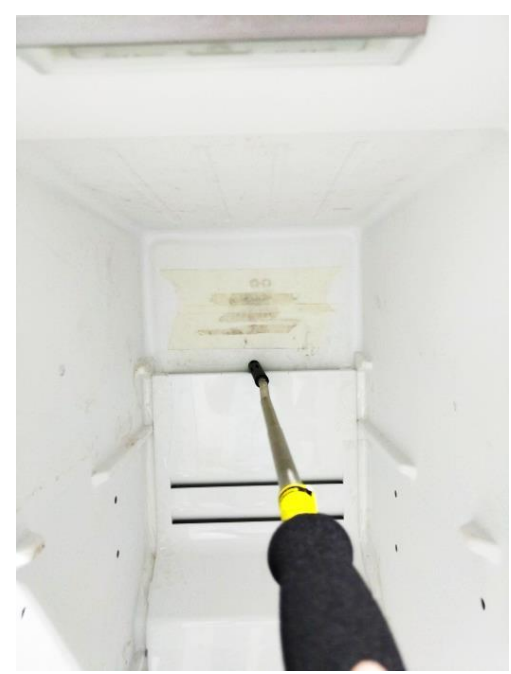

Fig. 4. Photograph of airflow velocity measurement experiment site. 


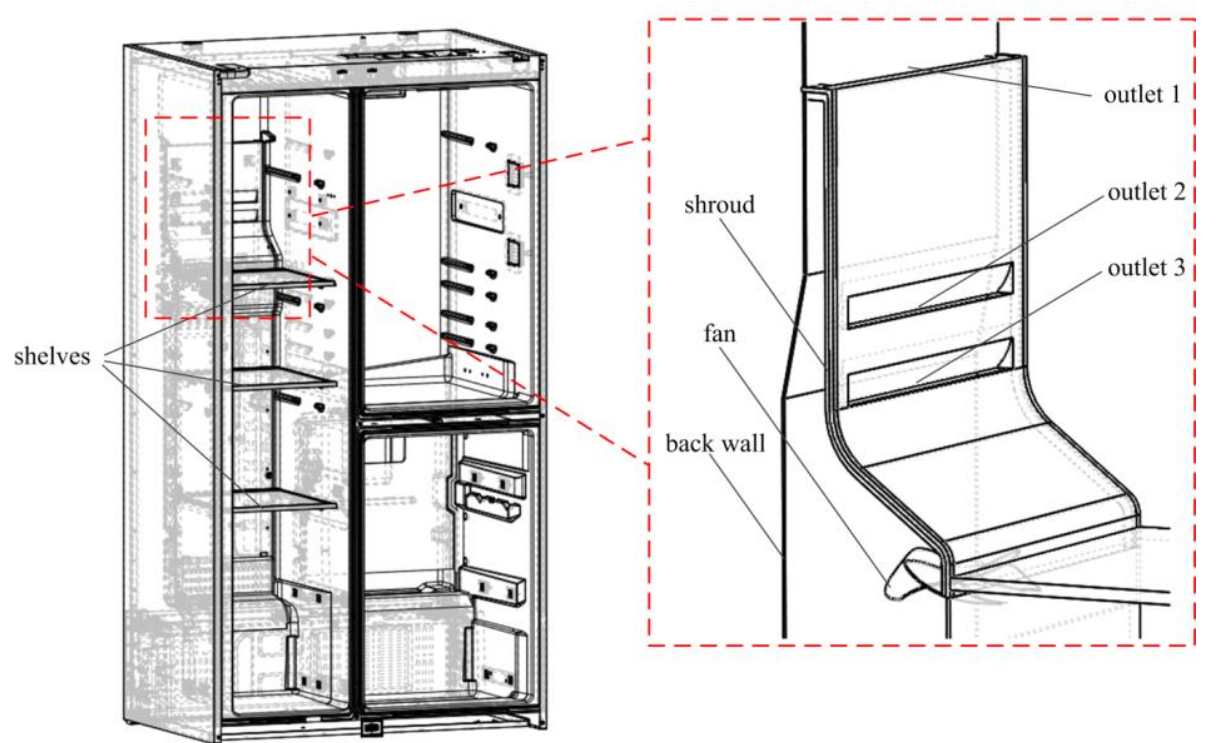

Fig. 2. Composition of the pneumatic system in freezer compartment.

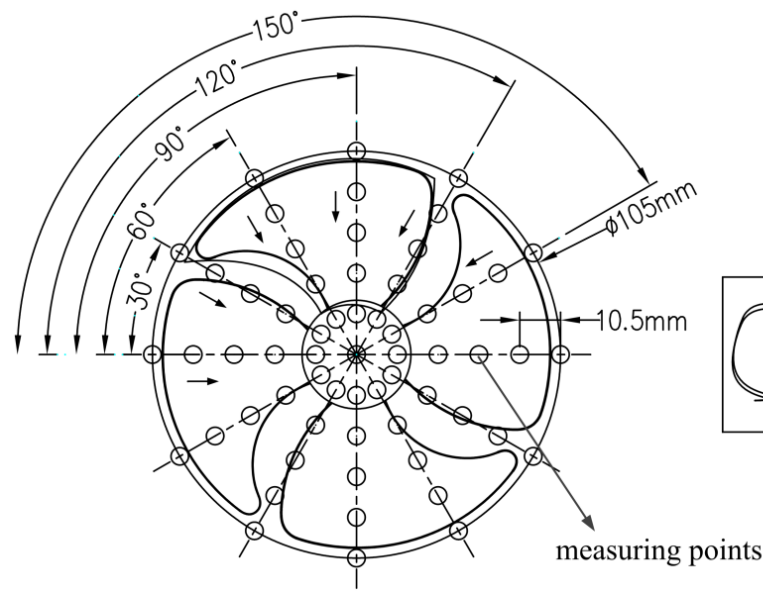

Fig. 3. Schematic diagram of measuring plane and measuring points.

Table 1 Errors between the second group and the fourth group data

\begin{tabular}{|c|c|c|c|c|c|c|c|c|c|c|c|}
\hline Point number & 1 & 2 & 3 & 4 & 5 & 6 & 7 & 8 & 9 & 10 & 11 \\
\hline Error $(\%)$ & 0 & 1.35 & 1.24 & 2.01 & 2.58 & 0 & 3.56 & 4.57 & 0.65 & 6.64 & 1.82 \\
\hline
\end{tabular}

When measuring the airflow velocity of points on each line, the measurement is performed in the order of the measurement point numbers from 1 to 11. The airflow velocities measured in the experiment are shown in Appendix A and errors between the six groups of data were calculated. The errors between the second group and the fourth group are the smallest, and the error calculation results of the second group and the fourth group are shown in Table 1 . It can be seen from Table 1 that the minimum error between the 11 points is 0 , the maximum error is $6.64 \%$, and others are all within $5 \%$, indicating that the airflow velocities at points corresponding to a certain point number on the fan have a high degree of circumferential uniformity.

The data in Appendix A is drawn into a threedimensional figure by the SRV fitting method, which is shown in Fig. 5. As can be seen from Fig. 5 , from the edge of the fan to the center of the hub, the airflow velocities increase first and then decrease and the airflow velocities at the edge and the center of the fan are smaller. The airflow velocity values at each group of points which have the same distance from the center of the hub have a high degree of circumferential uniformity. The fitted velocity values are used as the velocity entry boundary condition for the following CFD simulation by UDF method. 


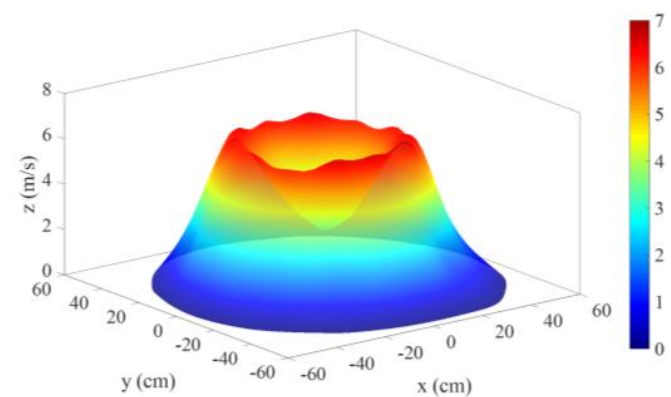

Fig. 5. Distribution of airflow velocities above the fan.

\section{CFD Simulations}

To evaluate the effects of the shroud and other critical parameters on the performance of the refrigerator, CFD simulations are performed; where by the details of the flow can be obtained. It should facilitate a direct understanding of the flow field and then is helpful for further design and optimizations. The details of the CFD simulation, including basic theory, flow domain determination, mesh grid generation, boundary setting, and solver setting are presented in this part.

\subsection{Basic Theory}

The essence of performing CFD simulation is to solve the partial differential governing equations based on designated boundaries. The governing equations refer to the conservation equations regarding the mass, momentum, and energy (Song et al. 2014). For all CFD simulations, these conservation equations are indispensable, as shown below.

$$
\frac{\partial \rho}{\partial t}+\nabla \cdot(\rho \vec{v})=S_{m}
$$

Momentum conservation Equation:

$$
\begin{aligned}
& \frac{\partial}{\partial t}(\rho \vec{v})+\nabla \cdot(\rho \vec{v} \vec{v})=-\nabla p+\nabla \cdot(\overline{\bar{\tau}})+\rho \vec{g}+\vec{F} \\
& \overline{\bar{\tau}}=\mu\left[\left(\nabla \vec{v}+\nabla \vec{v}^{T}\right)-\frac{2}{3} \nabla \cdot \vec{v} I\right]
\end{aligned}
$$

Where $\rho$ is the density, $\vec{v}$ is velocity, $S_{m}$ are items of source, $p$ is the static pressure, $\overline{\bar{\tau}}$ is the stress tensor, $\rho \vec{g}$ is the gravitational body force, $\vec{F}$ is external body forces, $\mu$ is the molecular viscosity and $I$ is the unit tensor.

Due to the high-nonlinear features of turbulence, to solve these differential equations directly is of great difficulties. To overcoming this, the RANS (Reynolds Averaged Navier-Stokes) method is adopted to isolate the time-averaged properties from the transient parts. This ignored the extreme transient flow features by inducing time-averaged items, but it results in the equation set unclosed. To close the equation set, the Boussinesq Eddy Viscosity is assumed and formulated in terms of the turbulent kinetic energy, $k$, and the turbulent dissipation rate, $\varepsilon$, the specific dissipation rate. $K$ and $\varepsilon$ require their own turbulent transport models (Song et al. 2010).

For the $k-\varepsilon$ turbulence model,

$\mu_{t}=\rho C_{\mu} \frac{k^{2}}{\varepsilon}$

Where $\mu_{t}$ denotes the eddy viscosity, $C_{\mu}$ denotes the experimental constant, $\rho$ denotes density.

\subsection{CFD Model Development}

To reproduce the flow field in actual operation, a 3$\mathrm{D}$ model is developed in this part. Performing CFD simulation requires the flow domain to be determined first. The flow domain determined in this paper is shown in Fig. 6. Since the flow domain has a symmetry feature, a half 3-D model is adopted for simulations.

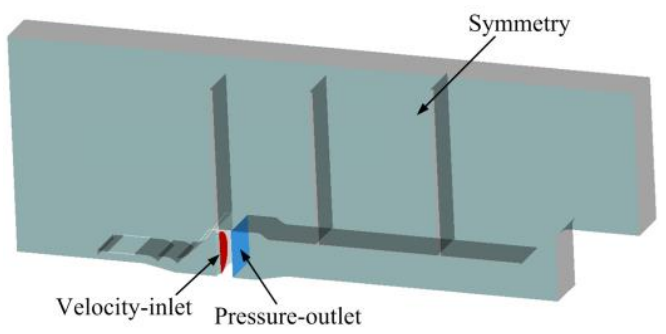

Fig. 6. Flow domain investigated in this paper.

Figure 7 shows the schematic of the determined flow domain, the inlet is defined at the positions near the front surface of fan, while the outlet is defined at the back of the fan. To facilitate mesh grid generation, the whole fluid domain is divided into three sub-domains, i.e., sub-domains of the chamber, fin, and outer.

Mesh girds are very important to ensure the accuracy and convergence of CFD simulations. To control the mesh quality and improve simulation efficiency, different mesh density levels are implemented for different sub-domains. In order to ensure the simulation is independent of the mesh grid, a series of mesh tests were carried out, the used mesh density and result of the mesh independence test is shown in Table 2 and Fig. 8, respectively. Since the flow distribution is the critical parameter for this study, the mass flow rate of the first fin gap is set as the compared parameter for mesh grid tests.

Table 2 Mesh density for mesh independence test

\begin{tabular}{|c|c|c|}
\hline Grid & \multicolumn{2}{|c|}{ Grid density $\left(\mathrm{cell} / \mathrm{mm}^{2}\right)$} \\
\hline & Average & Fin region \\
\hline Coarse & 0.11 & 1 \\
\hline Fine & 0.24 & 4 \\
\hline Very fine & 0.48 & 7 \\
\hline
\end{tabular}

It can be seen from Fig. 8 that a total of four twoequation turbulence models are adopted for mesh independence test, and similar results are obtained. 
X. F. Du et al. / JAFM, Vol. 14, No. 1, pp. 37-48, 2021.
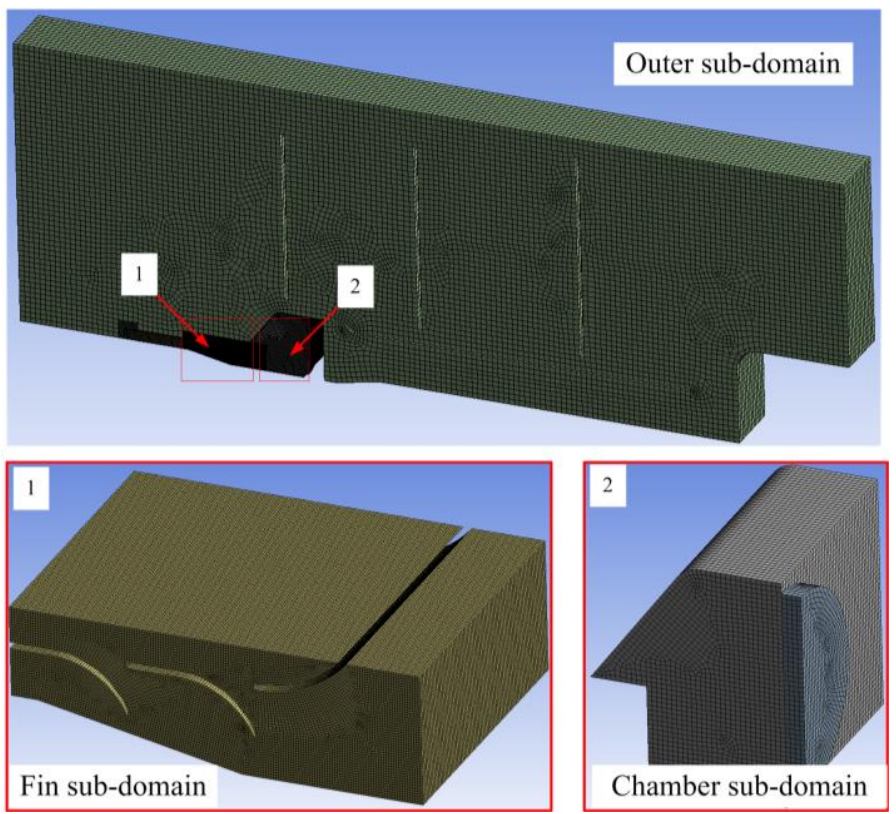

Fig. 7. Schematic of the determined flow domain.

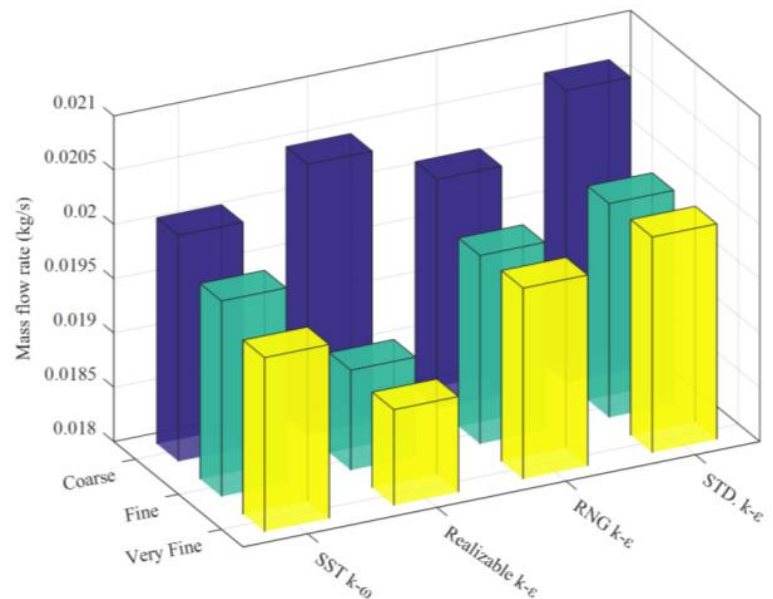

Fig. 8. Result of mesh independence test.

The mass flow rate obtained by Coarse and Fine mesh levels are different, while the difference between Fine and Very Fine is negligible. Thus, it can be inferred that when the mesh density is greater than Fine level, the simulation results do not change with the mesh grid any more, namely, the simulation result is of mesh independence. To ensure the accuracy and efficiency, the Fine mesh level is adopted for simulation in this study. In addition, Fig. 8 also indicate that the mass flow rate obtained from different turbulence models (with Fine and Very Fine meshes) are similar (maximum error $<5 \%$ ), which means that all of these models can accurately predict the mass flow rate. However, when performing simulation, the turbulence model of standard $\mathrm{k}-\varepsilon$ takes the least time, i.e., the most efficiency. Thus, in this paper, the standard $\mathrm{k}-\varepsilon$ model was implemented for simulations.

In addition to the mesh grid, the boundary conditions also have great influence on the CFD simulations. The boundary conditions specified in this paper is shown in Fig. 6. The inlet was specified as velocity-inlet, which is corresponding to the measured flow velocity in experimental tests. To convert the measured data to CFD simulations, the SRV method is adopted to fit the tested data, as shown in Fig. 5.

To assign the fitted velocity to CFD simulations, i.e., velocity-inlet, User Defined Methods (UDFs) was implemented, where the DEFINE_PROFILE macro was adopted to define the velocity-inlet velocity profile. The boundary of outlet was specified as pressure-outlet and the static pressure was set to be $0 \mathrm{~Pa}$.

\subsection{Solver Setting}

The CFD simulations are performed by using commercially available code ANASY Fluent 19.0. The solver settings for CFD simulations are shown in Table 3. 
Table 3 Details of solver setting

\begin{tabular}{|c|c|}
\hline Solver & Pressure-based, Steady \\
\hline Fluid & Gas with constant density \\
\hline Solution method & $\begin{array}{c}\text { Coupled scheme; Second } \\
\text { order }\end{array}$ \\
\hline Convergence criterion & $1 \mathrm{e}-5$ \\
\hline Turbulence models & Standard $k-\varepsilon$ \\
\hline
\end{tabular}

\subsection{Simulation Results}

The CFD simulation results of the freezer compartment are shown in Fig. 9. It can be seen from Fig. 9 a) that there is almost no streamline in the middle two cavities of the freezer compartment, indicating that the airflow velocity of the two cavities is very small, which will seriously affect the speed and effect of cooling. The airflow velocities are below $8 \mathrm{~m} / \mathrm{s}$ except for the shroud region. To obtain more details of the shroud region, the velocity vector in the shroud region is shown in Fig. 9 b). As can be seen from Fig. 9 b), the maximum velocity at the shroud region is 11.41 $\mathrm{m} / \mathrm{s}$, which appears near outlet 3 . At the same time, the velocities at the outlets 1,2 , and 3 show a certain difference, indicating that the uniformity of the airflow velocities of the three outlets of the existing shroud structure is not good.

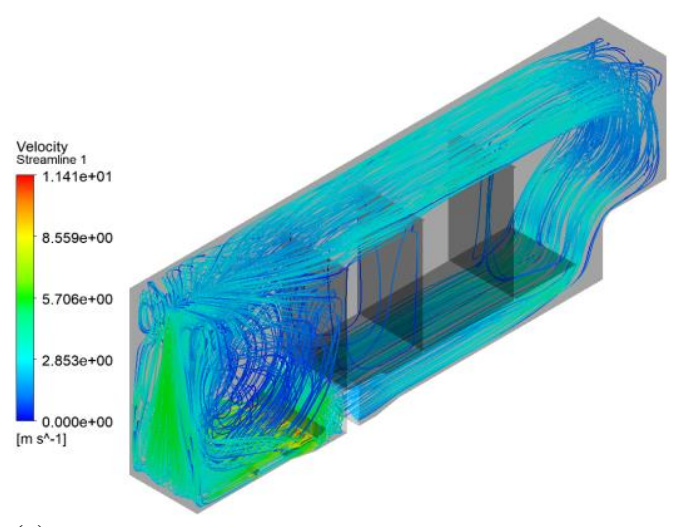

(a)

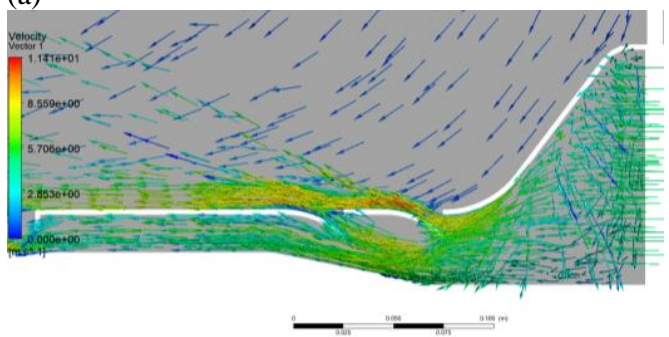

(b)

Fig. 9. CFD simulation results of the freezer compartment: (a) velocity streamline in the freezer compartment, (b) velocity vector in the shroud region.

Three straight lines are arranged near the outlets1, 2 , and 3 , and there are 50 monitoring points on each straight line. The airflow velocity data of these monitoring points was extracted after the CFD simulations, which is shown in Fig.10. Due to the symmetry of the fluid domain, half of the freezer compartment was selected during the simulation. Therefore, the airflow velocities shown in Fig. 10 are these of half the width near the three outlets, and those velocities at the other half width are symmetrical with Fig. 10.

It can be seen from Fig. 10 that the velocity at every monitoring point of outlet 3 is greater than outlet 1 and 2 , and the maximum velocity of monitoring points of outlet 1 is the smallest among the three outlets. The maximum velocities of outlets 2 and 3 appear at the center position, and the maximum velocity of the air outlet 1 appears at $1.15 \mathrm{~cm}$ from the center position. Meanwhile, it can be concluded that the airflow velocities at the edges of three outlets are smaller than those at the center position, among which the velocity attenuation of outlet 1 and 2 is significantly larger than that of outlet 3 .

To summarize, the velocity uniformity of the cavities of the freezer compartment and the three outlets in the shroud region is needed to be improved by using an efficient and accurate optimization method so that a faster cooling speed and a better cooling effect could be achieved.

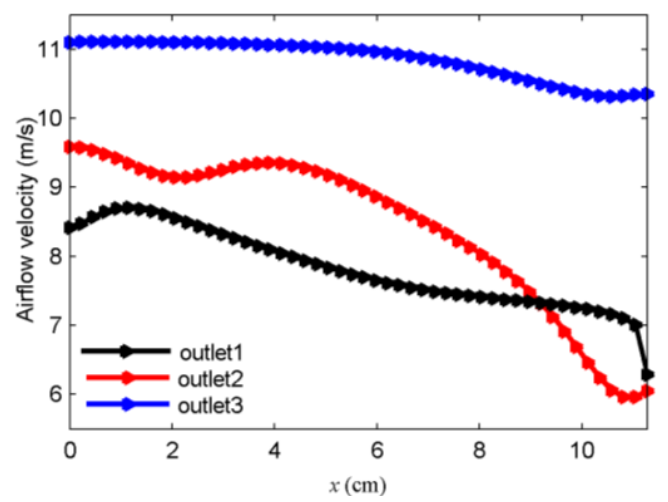

Fig. 10. Airflow velocities of three outlets.

\section{Parameter Optimization}

\subsection{Optimization Method}

To improve the uniformity of air flow in the freezing chamber, the key geometry parameters of the shroud and the distance between the shroud and other components such as the fan and shelves are optimized. There are many geometric parameters in the optimization, and the influence mechanism on the airflow velocities of three outlets and the airflow uniformity in the freezing compartment is also very complicated. In this paper, the BP neural network is used to establish an agent model for the simulation analysis of the flow field in the freezer compartment to improve the optimization efficiency. The agent model replaces the actual simulation model by fitting the sampling points in the design space and the construction process of the agent model is shown in Fig. 11. The design variables are 4 geometric parameters (as shown in Fig. 12), and the responses are the flow rate Q1, Q2, Q3 at the three outlets of the shroud. 


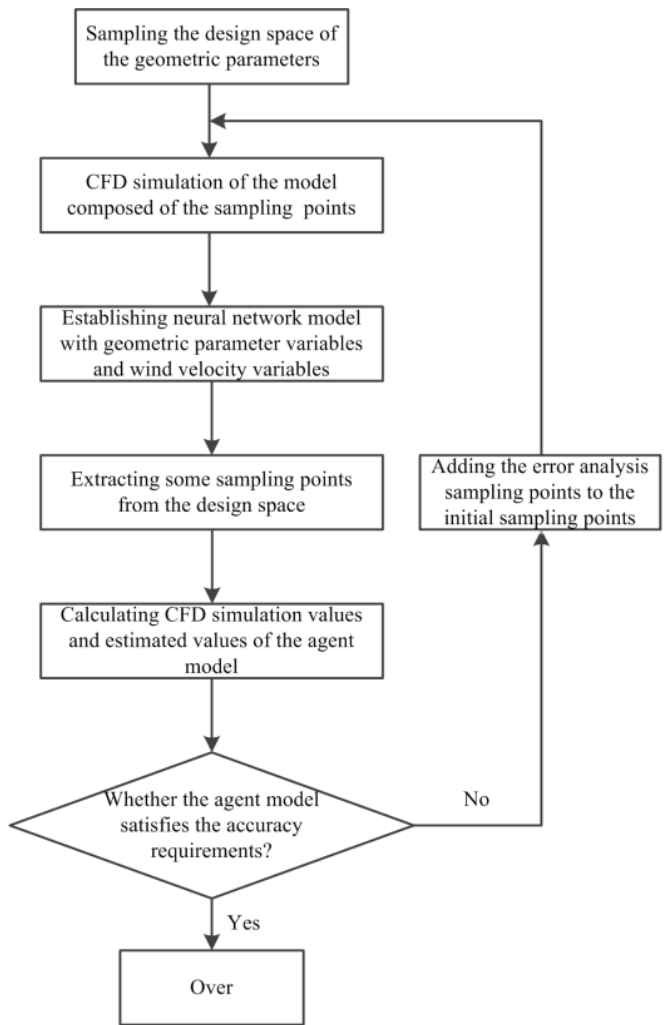

Fig. 11. Flow diagram of the agent model construction.

According to the simulation results and engineering experience, the values of $d_{1}$ and $R$ have a significant effect on the airflow distribution of the three air outlets. In addition, because the bend area of the shroud is located above the fan, the distance $h$ between the two components also affects the distribution of airflow near the shroud, and $d_{2}$ affects the flow of airflow in the compartments formed by the shelves. Therefore, the above four parameters are selected as the input layer. Besides, the flow rates of the three air outlets are large and have a large difference according to the simulation results. Compared with other points in the freezer compartment, when the four parameters of the input layer change, the flow rates at the three outlets are also more obvious, so selecting them as the output layer can accurately determine whether the result obtained by the agent model is accurate. The agent model is constructed by using the optimized Latin super-library method to extract 100 test points in the design space. Then, in the ISIGHT, the Creo, Workbench and Fluent are sequentially used to simulate the flow field of all the test points, and the response outputs under different design parameters are obtained. Finally, based on the above simulation results, the BP neural network is used in the ISIGHT to construct an agent model containing responses of all variables in the optimization.

There must be some error between the agent model and the actual value and the accuracy of the agent model can be evaluated by calculating the error. When the error analysis is performed, 30 sample data are randomly extracted in the design space, and the response outputs corresponding to the sample points can be calculated by the ISIGHT integrated model, and then compared with the calculation results of the agent model. The data types commonly used in the error analysis are maximum absolute error MAX, mean absolute error MAE, root mean square error RMSE and complex correlation coefficient $\mathrm{R}^{2}$. Among them, the RMSE and MAE mainly reflect the global error of the agent model, and the smaller the corresponding error value, the higher the global fitting accuracy of the agent model. The MAX reflects the local error of the agent model, and the smaller the corresponding error value, the higher the local fitting of the agent model. The complex correlation coefficient $\mathrm{R}^{2}$ is used to evaluate the degree of fitting of the agent model, and its value generally varies within the range of $[0,1]$. The more the value tends to 1 , the better the fitting degree of the agent model to the existing sample points and the stronger the ability to predict actual responses. In general, when RMSE and MAE are both less than 0.1, MAX $<0.4$, and $R^{2}>0.9$, the agent model can be considered to have high precision. The errors between the agent model and the CFD simulation results are calculated, as shown in Table 4. It can be seen from Table 4 that all the four parameters satisfy the above threshold requirements, so it can be considered that the agent model constructed in this paper has higher precision and can be used for subsequent optimization design.

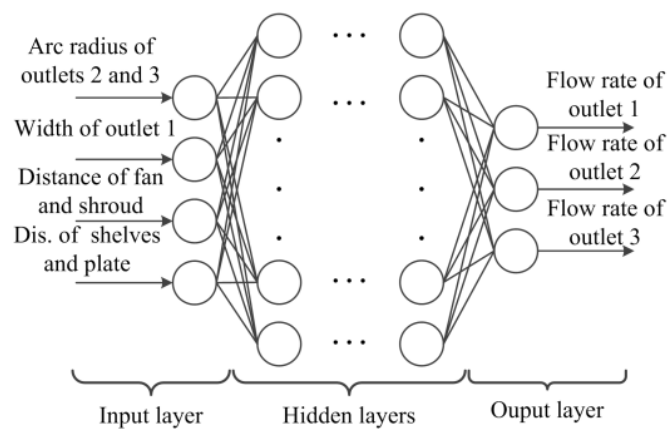

Fig. 12. Principle of the BP neural network.

Table 4 Agent model error analysis results

\begin{tabular}{|c|c|c|c|}
\hline Error term & Q1 & Q2 & Q3 \\
\hline MAE & 0.015 & 0.021 & 0.007 \\
\hline MAX & 0.042 & 0.055 & 0.196 \\
\hline RMSE & 0.038 & 0.036 & 0.022 \\
\hline $\mathrm{R}^{2}$ & 0.984 & 0.972 & 0.991 \\
\hline
\end{tabular}

The geometric dimensions to be optimized are shown in Fig. 13, where $R$ is the radius of the arcs at the outlets 2 and $3, d_{1}$ is the width of the outlet 1 , and $d_{2}$ is the distance between the edge of the shelves to the edge of the vertical board, and $h$ is the distance between the bottom surface of the fan rotating cylinder constructed during CFD simulation and the bottom surface of the shroud. The initial values of each parameter and the range of values when being optimized are shown in Table 5 . 


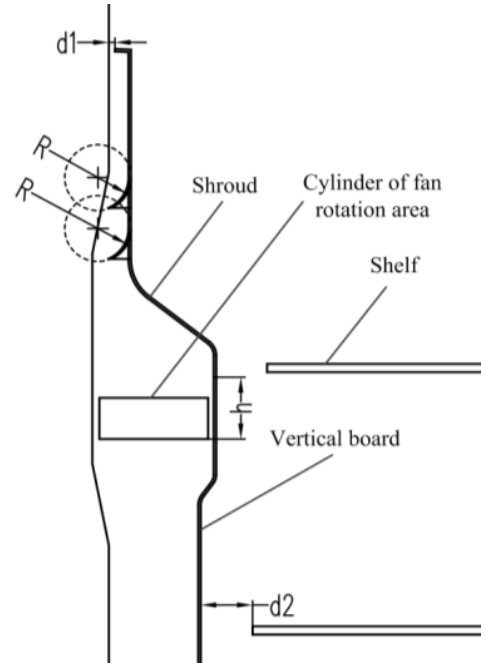

Fig. 13. Schematic diagram of structural geometric parameters to be optimized.

Table 5 Initial values and range of the geometric parameters to be optimized

\begin{tabular}{|c|c|c|c|}
\hline Variable & $\begin{array}{c}\text { Initial value } \\
(\mathrm{mm})\end{array}$ & $\begin{array}{c}\text { Lower limit } \\
(\mathrm{mm})\end{array}$ & $\begin{array}{c}\text { Upper limit } \\
(\mathrm{mm})\end{array}$ \\
\hline $\mathrm{R}$ & 41.5 & 30 & 50 \\
\hline $\mathrm{d}_{1}$ & 11 & 4 & 16 \\
\hline $\mathrm{d}_{2}$ & 0 & 0 & 50 \\
\hline $\mathrm{h}$ & 26.14 & 20 & 65 \\
\hline
\end{tabular}

The mathematical model of optimization is

$$
\min J_{1} * 0.4+J_{2} * 0.6
$$

Where $J_{l}$ is the mean square error of the airflow velocities of the three outlets.

$$
J_{1}=\sqrt{\left(v_{1}-v_{2}\right)^{2}+\left(v_{2}-v_{3}\right)^{2}+\left(v_{1}-v_{3}\right)^{2}} / 3
$$

$J_{2}$ is the mean square error of the airflow velocities at five points on the centerline of the freezer compartment.

$$
J_{2}=\sqrt{\begin{array}{l}
\left(v_{4}-v_{5}\right)^{2}+\left(v_{4}-v_{6}\right)^{2}+\left(v_{4}-v_{7}\right)^{2}+ \\
\left(v_{4}-v_{8}\right)^{2}+\left(v_{5}-v_{6}\right)^{2}+\left(v_{5}-v_{7}\right)^{2}+/ 10 \\
\left(v_{5}-v_{8}\right)^{2}+\left(v_{6}-v_{7}\right)^{2}+\left(v_{6}-v_{8}\right)^{2}+ \\
\left(v_{7}-v_{8}\right)^{2}
\end{array}}
$$

The locations of the monitoring points in $J_{l}$ and $J_{2}$ are shown in Appendix B. Where $J_{I}$ is the mean square error of airflow velocities at points 1,2 , and $3, J_{2}$ is the mean square error of airflow velocities at points $4,5,6,7$ and 8 . The design parameters, optimization goals, and constraints are defined in the optimization design module in ISIGHT, where the constraints are determined according to Eq. (5), the variable ranges are determined according to Table 5, and the optimization direction is min. The particle swarm optimization algorithm is used as the optimization algorithm. The parameters are set as follows: the number of particles is 20 , the inertia weight decreases linearly from 0.96 to 0.5 , and the maximum iteration is 2000 . The optimized parameters are shown in Table 6.

Table 6 Comparison of parameters within and without optimization

\begin{tabular}{|c|c|c|c|c|}
\hline Variable & $\mathrm{R}(\mathrm{mm})$ & $\mathrm{d}_{1}(\mathrm{~mm})$ & $\mathrm{d}_{2}(\mathrm{~mm})$ & $\mathrm{h}(\mathrm{mm})$ \\
\hline Initial & 41.5 & 11 & 26.24 & 0 \\
\hline Optimized & 32 & 6 & 30 & 60 \\
\hline
\end{tabular}

\subsection{Optimization Results}

The 3D model was re-established based on the optimized parameters and a new CFD analysis was carried out using the established 3D model. The simulation results of the optimized structure were shown in Fig. 14. It can be observed that from Fig. 14 that the velocity streamlines in the middle two cavities are significantly increased, which means that the cooling speed and cooling effect of these two cavities have been much better after optimization.

The values of Eq. (5), (6), and (7) within and without optimization were calculated, which are shown in Table 7. It can be seen that from Table 7 that $J_{1}$ is reduced by $84.45 \%$, indicating that the uniformity of velocities at three outlets has been significantly improved. Meanwhile, the mean square error of the other five monitoring points in the freezer compartment was reduced by $47.55 \%$, which can be concluded that the overall velocity uniformity in the freezer compartment is also improved. The value of the mathematical model constructed in optimization has a reduction proportion of $61.09 \%$ compared with that of the original CFD model.

Table 7 Comparison of mean square error function values within and without optimization

\begin{tabular}{|c|c|c|c|}
\hline Variable & $\mathrm{J}_{1}$ & $\mathrm{~J}_{2}$ & $\mathrm{~J}_{1} \times 0.4+\mathrm{J}_{2} \times 0.6$ \\
\hline Initial & 0.8187 & 0.9418 & 0.8925 \\
\hline Optimized & 0.1273 & 0.4939 & 0.3473 \\
\hline $\begin{array}{c}\text { Reduced } \\
\text { proportion }\end{array}$ & $84.45 \%$ & $47.55 \%$ & $61.09 \%$ \\
\hline
\end{tabular}

\section{Conclusion}

Many literatures related to refrigerator using CFD approaches have been reported. However, these investigations focus mainly on shelves or plateevaporator, etc. In this study, to evaluate the effects of the shroud and other critical parameters on the performance of refrigerator, the accuracy of different turbulence models for CFD simulations was investigated and a series of CFD models of the fluid domain in the freezer compartment of the refrigerator were constructed. It has been found that when the mesh density is greater than Fine level, the simulation results do not change with the mesh grid any more, that is, the simulation result is of mesh independence. An experimental rig was established to measure the velocities of the plane above the fan, and the measured data were fitted by 

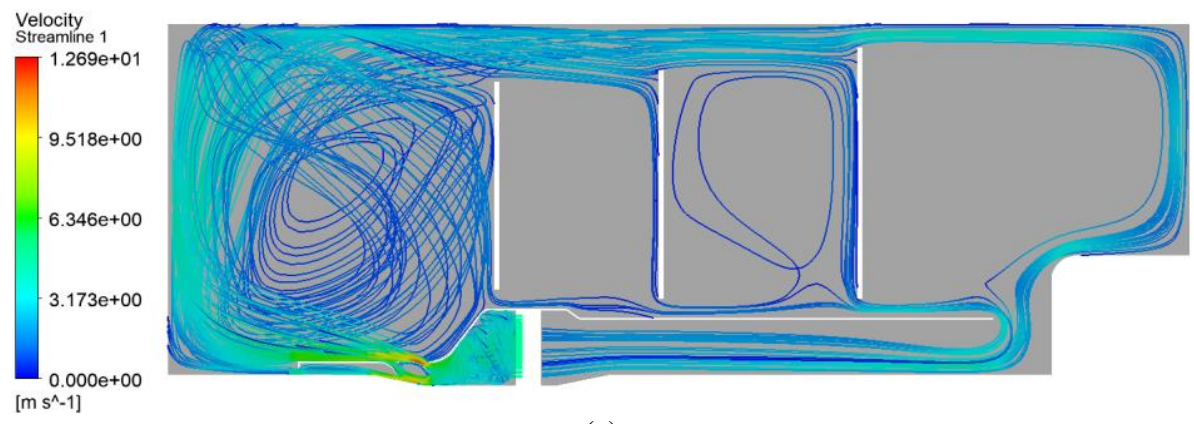

(a)

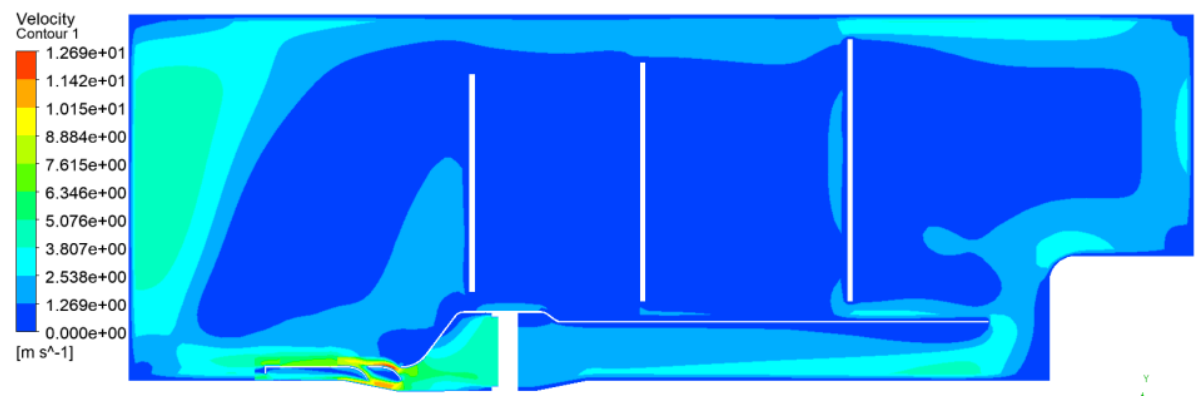

(b)

Fig. 14. Simulation results of the optimized structure: (a) Velocity streamline of the optimized structure, (b) Velocity contour of the optimized structure.

APPENDIX A: Experimental data of airflow velocity measurement.

\begin{tabular}{|c|c|c|c|c|c|c|c|c|c|c|c|c|}
\hline Times & $\begin{array}{c}\text { Position } \\
\text { angle }\left({ }^{\circ}\right)\end{array}$ & $\begin{array}{c}\text { Point 1 } \\
(\mathrm{m} / \mathrm{s})\end{array}$ & $\begin{array}{c}\text { Point 2 } \\
(\mathrm{m} / \mathrm{s})\end{array}$ & $\begin{array}{c}\text { Point 3 3 } \\
(\mathrm{m} / \mathrm{s})\end{array}$ & $\begin{array}{c}\text { Point 4 } \\
(\mathrm{m} / \mathrm{s})\end{array}$ & $\begin{array}{c}\text { Point 5 } \\
(\mathrm{m} / \mathrm{s})\end{array}$ & $\begin{array}{c}\text { Point 6 } \\
(\mathrm{m} / \mathrm{s})\end{array}$ & $\begin{array}{c}\text { Point 7 } \\
(\mathrm{m} / \mathrm{s})\end{array}$ & $\begin{array}{c}\text { Point 8 } \\
(\mathrm{m} / \mathrm{s})\end{array}$ & $\begin{array}{c}\text { Point 9 } \\
(\mathrm{m} / \mathrm{s})\end{array}$ & $\begin{array}{c}\text { Point } \\
10 \\
(\mathrm{~m} / \mathrm{s})\end{array}$ & $\begin{array}{c}\text { Point } \\
11 \\
(\mathrm{~m} / \mathrm{s})\end{array}$ \\
\hline 1 & 0 & 0.44 & 2.23 & 6.29 & 5.41 & 3.56 & 2.57 & 3.31 & 5.68 & 6.18 & 2.54 & 0.87 \\
\hline 2 & 30 & 0.49 & 2.97 & 6.46 & 5.48 & 3.49 & 2.27 & 3.93 & 5.03 & 6.20 & 2.41 & 0.55 \\
\hline 3 & 60 & 0.46 & 2.80 & 6.08 & 5.04 & 3.56 & 2.66 & 3.94 & 4.95 & 6.03 & 2.57 & 0.59 \\
\hline 4 & 90 & 0.49 & 2.93 & 6.54 & 5.37 & 3.58 & 2.27 & 3.79 & 4.80 & 6.24 & 2.25 & 0.56 \\
\hline 5 & 120 & 0.71 & 2.96 & 6.37 & 5.35 & 3.84 & 2.23 & 3.26 & 5.42 & 6.52 & 2.10 & 0.89 \\
\hline 6 & 150 & 0.44 & 2.22 & 6.19 & 5.86 & 3.86 & 2.30 & 3.86 & 5.41 & 6.43 & 2.21 & 0.47 \\
\hline
\end{tabular}

APPENDIX B: Schematic diagram of the location (in millimeters) of monitoring points.

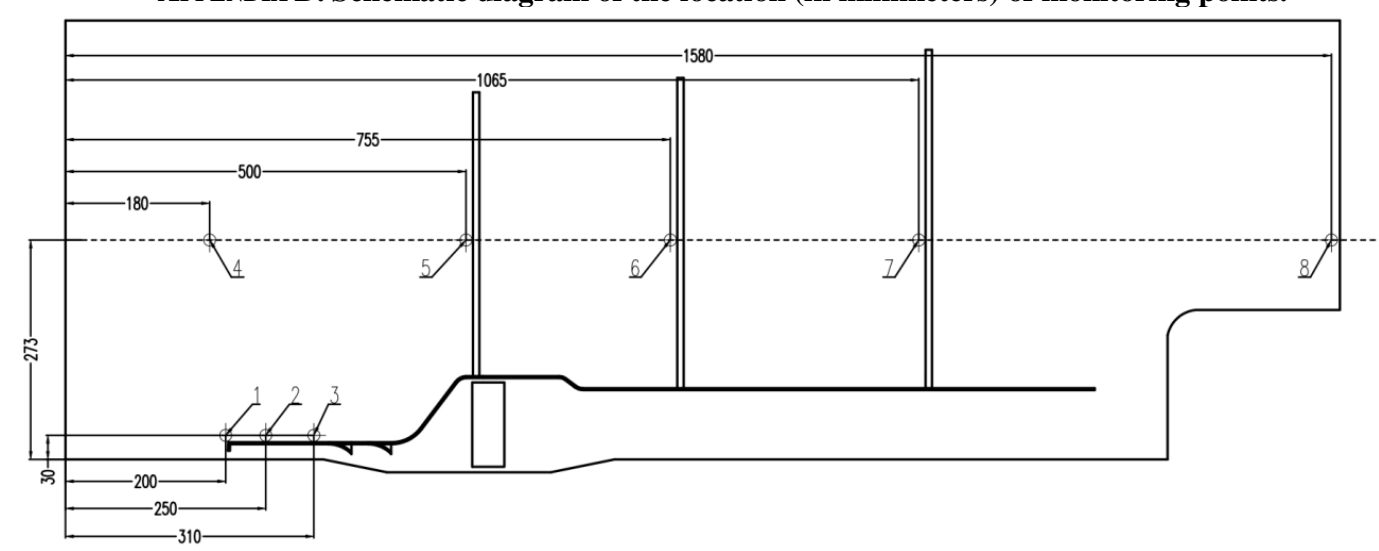

SRV method and then assigned to CFD simulations. Simulation results reveal that the maximum velocity at the shroud region is $11.41 \mathrm{~m} / \mathrm{s}$, and the velocities at the outlets 1,2 , and 3 show a certain difference. 
Meanwhile, the airflow velocities at the edges of three outlets are smaller than those at the center position, among which the velocity attenuation of outlet 1 and 2 is significantly larger than that of outlet 3 .

To improve the uniformity of air flow in the freezing chamber, an agent model containing responses of four critical parameters as design variables based on the BP neural network was established and the accuracy of the agent model was verified by the error analysis. The mathematical model of optimization for the shroud of the refrigerator was proposed, and a set of optimized parameters was obtained by the particle swarm optimization algorithm in ISIGHT. CFD simulations of the optimized model indicate that the velocity streamlines in the middle two cavities are significantly increased and the value of the mathematical model constructed in optimization has a reduction proportion of $61.09 \%$ compared with that of the original CFD model.

\section{ACKNOWLEDGEMENTS}

This study is supported by the Science and Technology Support Program of Jiangsu, China (no.BE2014133), the Prospective Joint Research Program of Jiangsu, China (no.BY2014127-01) and the Achievement Transformation Project of Nanjing, China (no. 201701213). The financial support is gratefully acknowledged.

\section{REFERENCES}

Abe, K., M. Nishida, A. Sakurai, Y. Ohya, H. Kihara, E. Wada and K. Sato (2005). Experimental and numerical investigations of flow fields behind a small wind turbine with a flanged diffuser. Journal of Wind Engineering and Industrial Aerodynamics 93(12), 951-970.

Abu-Thuraia, H., C. Aygun, M. Paraschivoiu and M. A. Allard (2018). Computational fluid dynamic analysis of roof-mounted vertical-axis wind turbine with diffuser shroud, flange, and vanes. Transactions of the Canadian Society for Mechanical Engineering 42(4), 404-415.

Aranake, A. C., V. K. Lakshminarayan and K. Duraisamy (2015). Computational analysis of shrouded wind turbine configurations using a 3-dimensional RANS solver. Renewable Energy 75, 818-832.

Avcı, H., D. Kumlutaş, Ö. Özer and M. Özşen (2016). Optimisation of the design parameters of a domestic refrigerator using CFD and artificial neural networks. International Journal of Refrigeration 67, 227-238.

Belman-Flores, J. M. and A. Gallegos-Muñoz (2016). Analysis of the flow and temperature distribution inside the compartment of a small refrigerator. Applied Thermal Engineering 106, 743-752.

Ding, G. L., H. T. Qiao and Z. L. Lu (2004). Ways to improve thermal uniformity inside a refrigerator. Applied Thermal Engineering 24(13), 1827-1840.

Duan, C., H. Fukushima, K. Segewa, T. Shibata and H. Fujii (2019). Improvement of Steam Turbine Stage Efficiency by Controlling Rotor Shroud Leakage Flows-Part II: Effect of Axial Distance Between a Swirl Breaker and a Rotor Shroud on Efficiency Improvement. Journal of Engineering for Gas Turbines and Power 141(4), 041002.

Elarem, R., S. Mellouli, E. Abhilash and A. Jemni (2017). Performance analysis of a household refrigerator integrating a PCM heat exchanger. Applied Thermal Engineering 125, 1320-1333.

$\mathrm{Hu}$, J. and G. Ding (2006). Effect of deflecting ring on noise generated by outdoor set of a splitunit air conditioner. International Journal of Refrigeration 29(3), 505-513.

Hu, X., S. Wen, Y. Gao, G. Xi, B. Khalighi and J. P. Johnson (2011). Experimental study on the effect of the shroud on the performance and flow field of an automotive cooling fan. Proceedings of the Institution of Mechanical Engineers, Part D: Journal of Automobile Engineering 225(5), 627-642.

Huang, H., Wang, Z. and Z. Liu (2017). Investigation of aerodynamic performance of small axial flow fan coupled with deflecting ring. Proceedings of the Institution of Mechanical Engineers, Part C: Journal of Mechanical Engineering Science 231(10), 1839-1848.

Jafari, S. A. and B. Kosasih (2014a). Analysis of the power augmentation mechanisms of diffuser shrouded micro wind turbine with computational fluid dynamics simulations. Wind and Structures 19(2), 199217.

Jafari, S. A. and B. Kosasih (2014b). Flow analysis of shrouded small wind turbine with a simple frustum diffuser with computational fluid dynamics simulations. Journal of Wind Engineering and Industrial Aerodynamics 125, 102-110.

Jiang, L., X. Wu and Z. Zhang (2014). Conjugate Heat Transfer of an Internally Air-Cooled Nozzle Guide Vane and Shrouds. Advances in Mechanical Engineering 6, 146523.

Jung, U. H., S. Kim, K. H. Jeong, J. H. Kim, K. Y. Lee and Y. S. Choi (2016). Numerical study on performance improvement when strong vortex occurs on the shroud of vaneless diffuser in turbo blower. Journal of Mechanical Science and Technology 30(6), 2515-2529.

Mansouri, Z., A. Settar and H. Khamane (2018). Computational investigation of heat load and secondary flows near tip region in a transonic turbine rotor with moving shroud. Applied Thermal Engineering 136, 141-151. 
Neal, D. R. and J. F. Foss (2007). The application of an aerodynamic shroud for axial ventilation fans. Journal of Fluids Engineering 129(6), 764-772.

Pan, G., Y. Chang and M. Fu (2018). Computational fluid dynamics analysis of shroud design on hemodynamic performance and blood damage in a centrifugal blood pump. Computer Modeling in Engineering \& Sciences 116(2), 199-213.

Ren, G., S. Heo, T. H. Kim and C. Cheong (2013). Response surface method-based optimization of the shroud of an axial cooling fan for high performance and low noise. Journal of Mechanical Science and Technology 27(1), 3342.

Shibata, T., H. Fukushima K. Segewa (2019). Improvement of Steam Turbine Stage Efficiency by Controlling Rotor Shroud Leakage Flows-Part I: Design Concept and Typical Performance of a Swirl Breaker. Journal of Engineering for Gas Turbines and Power 141(4), 041003.

Song, X. G., L. Wang and Y. C. Park (2010). Transient analysis of a spring-loaded pressure safety valve using computational fluid dynamics (CFD). Journal of Pressure Vessel Technology 132(5), 054501.

Song, X., L. Cui, M. Cao, W. Cao, Y. Park and W. M. Dempster (2014). A CFD analysis of the dynamics of a direct-operated safety relief valve mounted on a pressure vessel. Energy Conversion and Management 81, 407-419.

Wadia, A. R. and P. N. Szucs (2008). Inner workings of shrouded and unshrouded transonic fan blades. Journal of Turbomachinery 130(3), 031010.

Wu, Chengjun, J. Liu, and J. Pan (2014). Influence of surrounding structures upon the aerodynamic and acoustic performance of the outdoor unit of a split air-conditioner. Chinese Journal of Mechanical Engineering 27(4) 836845.

Yalcin, H. G., S. Baskaya and M. Sivrioglu (2008) Numerical analysis of natural convection heat transfer from rectangular shrouded fin arrays on a horizontal surface. International Communications in Heat and Mass Transfer 35(3), 299-311.

Zhang, C. and Y. Lian (2014). Conjugate heat transfer analysis using a simplified household refrigerator model. International Journal of Refrigeration 45, 210-222.

Zhou, L., W. Shi, W. Li and R. Agarwal (2013). Numerical and experimental study of axial force and hydraulic performance in a deep-well centrifugal pump with different impeller rear shroud radius. Journal of Fluids Engineering 135(10), 104501. 